ARTICLE

Received 8 Aug 2014 | Accepted 19 Dec 2014 | Published 19 Feb $2015 \quad$ DOl: 10.1038/ncomms7165

\title{
Unfolding a molecular trefoil derived from a zwitterionic metallopeptide to form self-assembled nanostructures
}

Ye Zhang ${ }^{1}$, Ning Zhou' ${ }^{1}$ Junfeng Shi ${ }^{1}$, Susan Sondej Pochapsky¹, Thomas C. Pochapsky ${ }^{1}$, Bei Zhang ${ }^{2}$, Xixiang Zhang ${ }^{2} \&$ Bing $X u^{1}$

While used extensively by nature to control the geometry of protein structures, and dynamics of proteins, such as self-organization, hydration forces and ionic interactions received less attention for controlling the behaviour of small molecules. Here we describe the synthesis and characterization of a novel zwitterionic metallopeptide consisting of a cationic core and three distal anionic groups linked by self-assembling peptide motifs. 2D NMR spectra, total correlated spectroscopy and nuclear Overhauser effect spectroscopy, show that the molecule exhibits a three-fold rotational symmetry and adopts a folded conformation in dimethyl sulfoxide due to Coulombic forces. When hydrated in water, the molecule unfolds to act as a self-assembling building block of supramolecular nanostructures. By combining ionic interactions with the unique geometry from metal complex and hydrophobic interactions from simple peptides, we demonstrate a new and effective way to design molecules for smart materials through mimicking a sophisticated biofunctional system using a conformational switch.

\footnotetext{
${ }^{1}$ Department of Chemistry, Brandeis University, Waltham, Massachusetts 02454, USA. ${ }^{2}$ Nano-fabrication, Imaging \& Characterization Core Lab, King Abdullah University of Science and Technology, Thuwal 23955-6900, Saudi Arabia. Correspondence and requests for materials should be addressed to B.X. (email: bxu@brandeis.edu).
} 
P rotein folding ${ }^{1-4}$ is an essential step in generating a functional protein. Partial unfolding of natively folded proteins often results in fibrillar structures or filaments that play important roles in either normal physiological functions ${ }^{5}$ or pathological states 6,7 . For example, Pepys et al. ${ }^{8}$ discovered that amyloidogenic intermediates of lysozyme consist of a partially unfolded conformers. Hurle et al. ${ }^{9}$ have shown that the conversion of an immunoglobulin light chain into amyloid proceeds via partial unfolding under acidic conditions. Recently, partially unfolding $\alpha$-lactalbumin has found application in cancer therapy ${ }^{10}$. Although these proteins differ in sequence and native folds, their fibrils are morphologically and structurally similar, implying similar mechanisms of unfolding and fibrillation. These studies, in addition to providing useful insights for understanding pathogenic fibrillogenesis and contributing to the development of potential therapeutics, also offer fundamental guiding principles for designing soft materials as a new class of biomimetics ${ }^{11-15}$.

The structures of amyloid fibrils have been determined in atomic details ${ }^{16-20}$, the partial unfolding of proteins to form fibrillar structures exhibits several distinctive common features. First, the folding-unfolding transition requires a flexible molecular architecture. Second, the polypeptide backbone and hydrophobic side chains are largely buried within the folded structure. Partial unfolding exposes the hydrophobic groups, and fibrillation provides an alternative means of sequestering those groups from solvent. Third, the forces governing protein conformation include those resulting from intramolecular (hydrogen bonding and salt bridge formation), and intermolecular (solvent-solvent and solvent-protein) interactions. Both types of interactions depend on the specific structure of the protein and on the nature of the solvent ${ }^{21-23}$. Changing the nature of the solvent can change the balance between the driving forces for folding versus unfolding $22-24$, potentially destabilizing the native folded structure ${ }^{24,25}$, and initiating the self-assembly of fibrillar structures. The fibrillation conditions for a wide range of proteins, correlates with charge, secondary structure and hydration. Despite being well-established in the field of protein structures ${ }^{26}$ or applied in design of hybrid system and crystalline materials ${ }^{27-30}$, these guiding principles received less attention in the design of soft materials made of small molecules. Thus, we aim to apply these interactions for designing biomimetic materials.

Here we describe the synthesis and characterization of a trefoil zwitterioinic metallopeptide designed to mimic the folding/ unfolding behaviour of proteins. The hydration of the ionic species separates the charges in the zwitterions, and results in the unfolding of the trefoil, and by removing the hydration force the trefoil folds back. Such a folding-unfolding conformational transition leads to the exposure of the hydrophobic arms of the trefoil molecule, thus favouring intermolecular self-assembly in its unfolded conformation. Furthermore, we find that the selfassembly of this trefoil molecule is $\mathrm{pH}$ responsive. The $\mathrm{pH}$ of the solvent modulates the trefoil molecule's hydration, which, in turn, is implicated in interfibrillar interactions that control filament morphology. This simple multimodular molecular system mimics protein unfolding in precise molecular details, and opens new possibilities for developing simple small molecules that mimic the behaviour and ultimately the functions of the complex biological systems.

\section{Results}

Molecular design. Figure 1 shows the concept of molecular design. The molecule consists of three modules, a dicationic metal complex as the positively charged core, three tripeptide arms ${ }^{31}$ capped by sulfonate groups as the distal anions ${ }^{32}$. To generate a

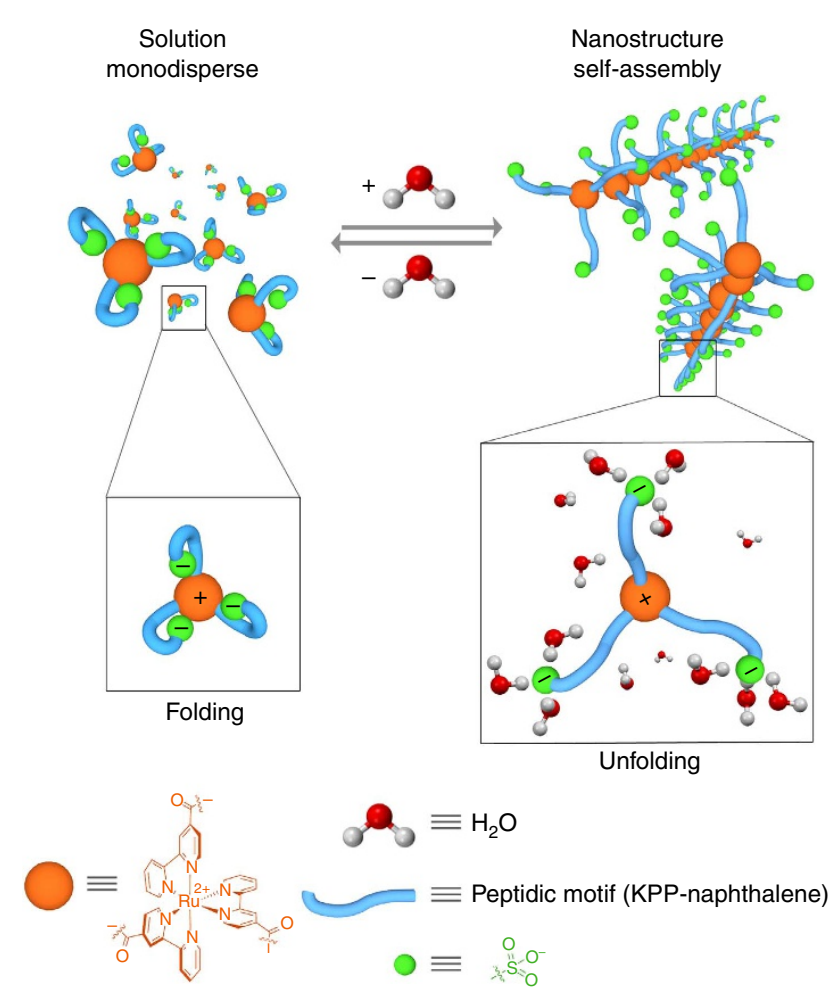

Figure 1 | Reversible unfolding of a trefoil molecule by hydration force for self-assembly. A trefoil molecule, consisting of a positive charged core (that is, $\left[\mathrm{Ru}(\mathrm{II})(\mathrm{bpy})_{3}\right]^{2+}$ analogue) and three negative charged groups (that is, sulfonate) that are covalently linked by three peptidic arms LysPhe-Phe-naphthalene (KPP-naphthalene), exists as a folded monomer in DMSO due to self-charge compensation (for clarity, $\mathrm{Na}^{+}$is not shown). The addition of water hydrates the ions in the trefoil molecule and unfolds it to allow the formation of supramolecular nanofibrils through intermolecular self-assembly. By decreasing the percentage of water using blowing air, nanofibrils dissociate. The trefoil molecules fold back and stay as monomers.

molecule capable of forming a three-dimensional folded structure, we choose an octahedral $\left[\mathrm{Ru}(\mathrm{II})(\mathrm{bpy})_{3}\right]^{2+}$ complex $^{33}$ as the core because ruthenium (II) also has a high affinity for sulfonate ${ }^{34}$. Since Hunter and colleagues successfully used octahedral $\left[\mathrm{Zn}(\mathrm{II})(\mathrm{bpy})_{3}\right]^{2+}$ to create a molecular trefoil $\mathrm{knot}^{35}$, the $\left[\mathrm{Ru}(\mathrm{II})(\mathrm{bpy})_{3}\right]^{2+}$ should also permit the formation of an open trefoil. To ensure the flexibility of the tripeptide arms for the folding-unfolding switch, we connected the metal complex to the peptide through the side chain of the lysine residue rather than the peptide main chain. To allow sufficient separation of the anions and the cationic core, we arrange the sulfonate groups at the end of the peptidic backbone. Overall, this molecule possesses a three-fold rotational symmetry and relies on the balance between ionic interactions and hydration force to determine the preferred molecular conformation. We expected that this arrangement would facilitate a conformational switch when the dielectric constant of the solvent changes.

Synthesis of trefoil zwitterionic metallopeptide. According to the molecular design, we synthesize the trefoil molecule $\mathbf{5}$ in three primary steps as presented in Fig. 2. We first prepare the peptide motif 1 using solid-phase peptide synthesis. On peptide motif $\mathbf{1}$, $N$-tert-butoxycarbonyl (Boc) group protects the $\varepsilon$-amino group of the lysine residue to facilitate the direct coupling of taurine to the peptide main chain. Following the coupling reaction, $N$-Boc deprotection in trifluoroacetic acid/dichloromethane mixture 


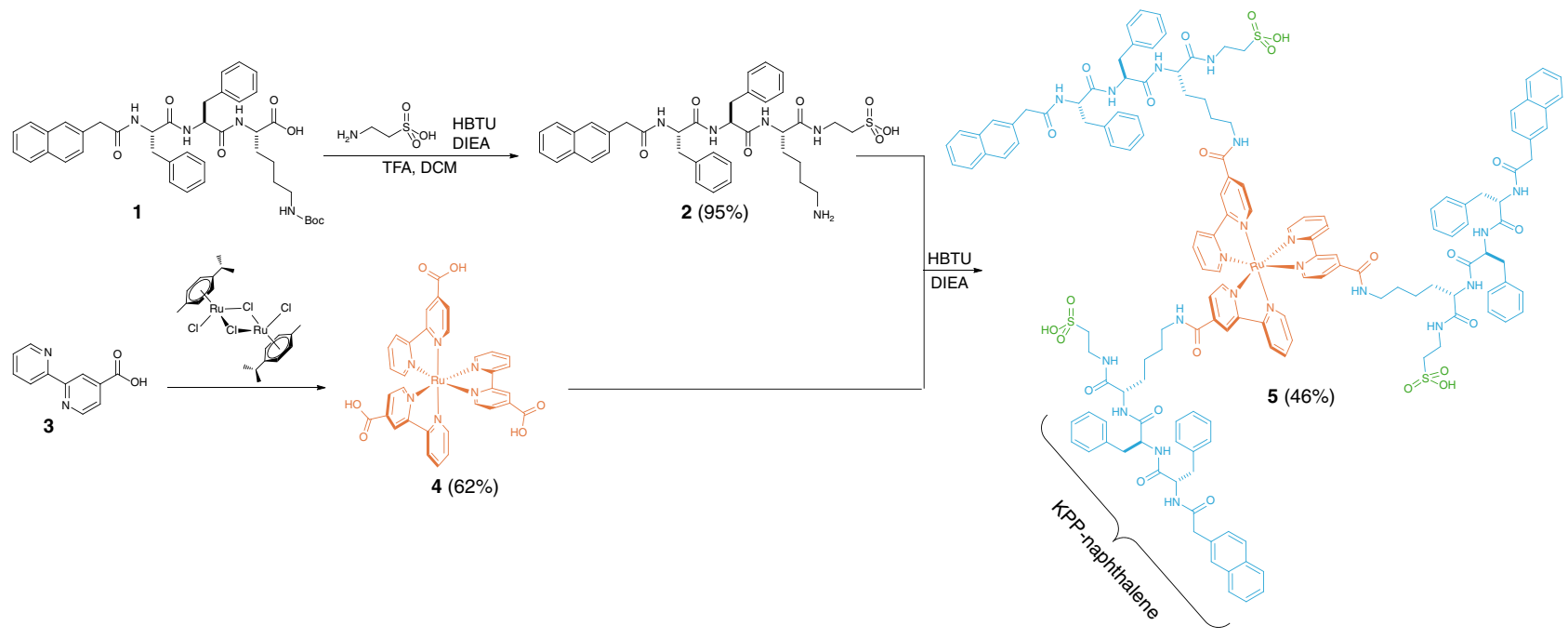

Figure 2 | Synthesis of the trefoil complex $\mathbf{5}$ based on octahedral-coordinated Ru(II). Compound $\mathbf{1}$ is coupled with taurine followed by removal of the Boc protecting group with trifluoroacetic acid in dichloromethane to give $\mathbf{2}$. The reaction of $\mathbf{3}$ with dichloro( $p$-cymene)Ru(II) dimer produces complex $\mathbf{4}$. Being assisted by HBTU and DIEA in anhydrous DMF, the coupling reaction of $\mathbf{2}$ and $\mathbf{4}$ affords the trefoil complex $\mathbf{5}$ (the metal complex core is represented in orange, the peptidic motif KPP-naphthalene in blue, and the sulfonate in green).

affords compound 2, the peptide with sulfonate group at its C-terminal, in $95 \%$ yield. An organometallic chelation of the bipyridine ligand (3) and dichloro( $p$-cymene) $\mathrm{Ru}(\mathrm{II})$ dimer in dimethylformamide (DMF) under reflux condition produces octahedral Ru-complex $\mathbf{4}$ as the positive charged core. After purification by Sephadex column chromatography, we obtain pure complex 4 as a red powder in a $62 \%$ yield. It takes $24 \mathrm{~h}$ to complete the final step of the synthesis - the direct coupling of $\mathbf{2}$ and 4 assisted by HBTU (O-(benzotriazol-1-yl)- $N, N, N^{\prime}$, $N^{\prime}$-tetramethyluronium hexafluorophosphate) and DIEA $(N$, $\mathrm{N}$-diisopropylethylamine) in DMF at $60^{\circ} \mathrm{C}$. Following a two-step purification, Sephadex column chromatography and semipreparative HPLC, we obtain the trefoil molecule $\mathbf{5}$ as an orange powder in $46 \%$ yield.

Folded structure. We investigate the structure of trefoil molecule 5 in dimethylsulphoxide (DMSO; $4.0 \mathrm{mM}$ ) using $1 \mathrm{D}^{1} \mathrm{H}$ nuclear magnetic resonance $\left({ }^{1} \mathrm{H} \mathrm{NMR}\right)$, and $2 \mathrm{D}{ }^{1} \mathrm{H}$-total correlation spectroscopy (TOCSY) and ${ }^{1} \mathrm{H}$-nuclear Overhauser effect spectroscopy (NOESY). The combination of $1 \mathrm{D}{ }^{1} \mathrm{H} \quad \mathrm{NMR}$ (Supplementary Fig. 1) and 2D ${ }^{1} \mathrm{H}$-TOCSY (Supplementary Fig. 2) data provides the complete ${ }^{1} \mathrm{H}$ NMR resonance assignments (Supplementary Table 1). $800 \mathrm{MHz}{ }^{1} \mathrm{H} \quad \mathrm{NMR}$ (Supplementary Fig. 1) provides sufficient dispersion of the $\mathrm{NH}$ signals $\left(\mathrm{W}_{1}, \mathrm{~W}_{2}, \mathrm{~W}_{4}\right.$ and $\mathrm{W}_{7}$ in the region of 7.92-8.27 p.p.m.) and splittings of $\mathrm{C}-\mathrm{H}$ signals $\left(\mathrm{X}_{2}\right.$, quartet, $\mathrm{X}_{4}$ and $\mathrm{X}_{7}$ are triplets of doublets), simplifying resonance assignments. The protons $1 \mathrm{a}$ and $1 \mathrm{~b}$ are assigned on the basis of ${ }^{1} \mathrm{H}$-TOCSY cross peaks with the $\mathrm{W}_{1}$ proton. The protons $3 \mathrm{a}, 3 \mathrm{~b}$ and $3 \mathrm{c}$ are assigned on the basis of cross peaks with the $\mathrm{W}_{2}$ and $\mathrm{X}_{2}$ protons. Following that, the proton $3 \mathrm{~d}$ is assigned based on the cross peak with $3 \mathrm{a}, 3 \mathrm{~b}$ and $3 \mathrm{c}$ protons. On the basis of the cross peak with the $3 \mathrm{~d}$ proton, the proton $\mathrm{W}_{3}$ is assigned. The combination of chemical shifts, peak splits, and cross peaks allows the assignment of protons $5 b, 6 b, 6 c$, $6 \mathrm{~d}, 6 \mathrm{e}$ and $8 \mathrm{a}$. On the basis of the cross peak with $8 \mathrm{a}$, proton $\mathrm{Z}_{8}$ is assigned. The remaining aromatic $\mathrm{C}-\mathrm{H}$ protons are assigned to the region between 7.29-7.13 p.p.m.

After completing the assignments of protons, we identified the nuclear Overhauser effects (NOEs; Supplementary Table 1, Supplementary Figs 2 and 3) to use as distance restraints for structural calculations. By applying energy minimization in
Chem3D, we build up the 3D structure of $\mathbf{5}$ based on the crystal structure of $\left[\mathrm{Ru}(\mathrm{II})(\mathrm{bpy})_{3}\right]^{2+}$ derivative ${ }^{36}$ and observed NOEs for 5 . NOEs between $3 a / 1 b, 3 d / 1 a, 3 d / 1 b, W_{1} / 3 a, W_{1} / 3 b$ and $\mathrm{W}_{1} / \mathrm{X}_{2}$ indicate the proximity between the taurine residues and the side chain of lysine residues on the trefoil molecule. In particular, the NOEs between $1 \mathrm{a}, 1 \mathrm{~b}$ and $3 \mathrm{~d}$ restrict the relative conformations of these residues. We assigned the phenylalanine main chain adjacent to lysine residue based on the NOE between $\mathrm{W}_{4}$ and $1 \mathrm{~b}$. The NOE between $\mathrm{Z}_{4}$ and $\mathrm{W}_{7}$ restricts the main chain conformation of the second phenylalanine residue. Finally, the NOE between $Z_{8}$ and $W_{4}$ restricts the conformation of the acetyl naphthalene moiety. The cross peaks between the aromatic $\mathrm{C}-\mathrm{H}$ protons in the region of $8.30-7.30$ p.p.m. define the relative positions of all the aromatic rings of the molecule. Figure $3 \mathrm{e}$ (stick model), 3f (stick model without protons) and $3 \mathbf{g}$ (space-filling model) show a 3D structure of trefoil 5 calculated by energy minimization that satisfies all distance restraints. Clearly, molecule 5 adapts a folded structure in DMSO. Since the low $\mathrm{pK}_{\mathrm{a}}$ (1.5) of taurine's sulfonic acid group ensures the moiety is negatively charged in neutral condition, this folded structure is likely due to the three sulfonate anions remaining in close proximity to the positive charged ruthenium centre.

Unfolded structure. To explore the structure of the trefoil molecule 5 under self-assembling conditions, we performed 1D ${ }^{1} \mathrm{H}$ NMR, 2D ${ }^{1} \mathrm{H}$-TOCSY and ${ }^{1} \mathrm{H}$-NOESY of the molecule in DMSO/ $\mathrm{D}_{2} \mathrm{O}(2 / 1)$ mixture $(4.0 \mathrm{mM})$. Note that the NMR signals are only slightly broader under the modified solvent conditions, so the observed species in NMR are not polymeric. However, the fluorescence scattering of $\mathrm{DMSO} / \mathrm{D}_{2} \mathrm{O}$ solution of 5 (Fig. $4 \mathrm{a}$; the ultraviolet light is placed on the right side of the NMR tube) indicates the formation of aggregates. This is confirmed by the high-resolution transmission electron microscopy (HRTEM) image of the dispersion that reveals uniform fibrils with widths of $4.5 \pm 0.1 \mathrm{~nm}$ (Fig. 4b). In the $1 \mathrm{D}{ }^{1} \mathrm{H}$ NMR spectrum (Supplementary Fig. 4), all $\mathrm{NH}$ protons disappear because of $\mathrm{D}_{2} \mathrm{O}$ exchange. Comparison of the $2 \mathrm{D}{ }^{1} \mathrm{H}$-TOCSY (Supplementary Fig. 5) and ${ }^{1}$ H-NOESY spectra (Supplementary Fig. 6) shows fewer inter-residue NOEs (Fig. 4d, Supplementary Table 2) of $\mathbf{5}$ under self-assembly conditions. While the lack of NOE-based distance restraints does not rule out the existence of folded 

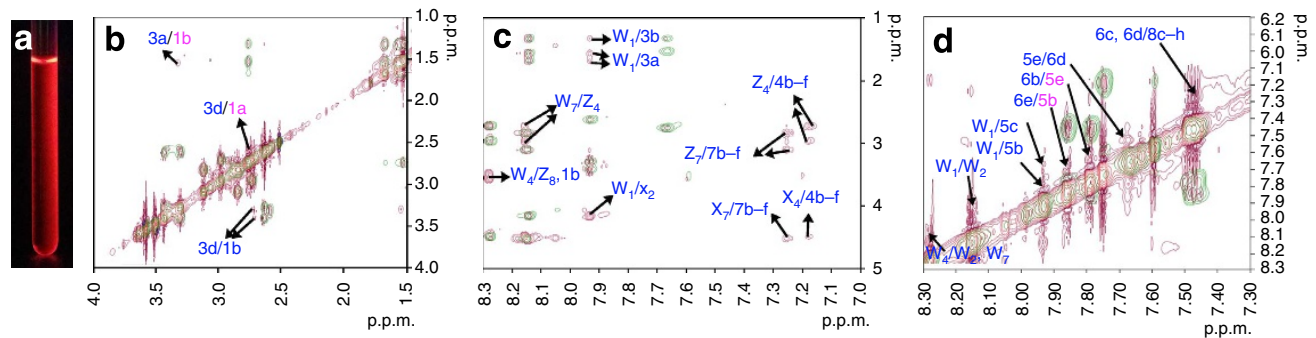

e
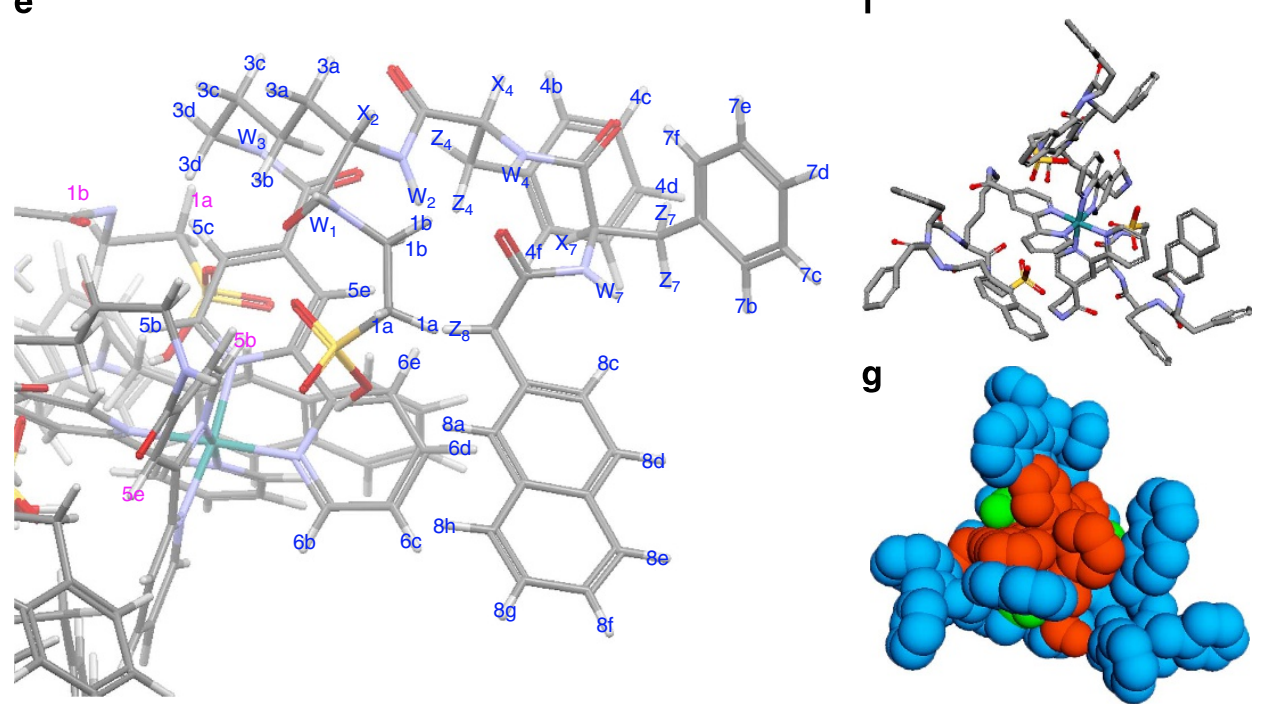

Figure 3 | 3D molecular structure of $\mathbf{5}$ in DMSO based on 2D NMR spectra. (a) Optical image of $\mathbf{5}$ in DMSO- $d_{6}$ in NMR tube under Ultraviolet light (on the right of the NMR tube). Overlapped spectra of ${ }^{1} \mathrm{H}$-TOCSY (green) and ${ }^{1} \mathrm{H}-\mathrm{NOESY}$ (red) (b), (c) and (d) assigned with NOEs. (e) Assignment of protons on a stick model of $\mathbf{5}$ in DMSO (inter-ligand NOEs are signed in magenta). (f) Full stick model and (g) space-filling model (colour representations of different portion of the molecule corresponding to the chemical structure in Fig. 2) of $\mathbf{5}$ in DMSO.
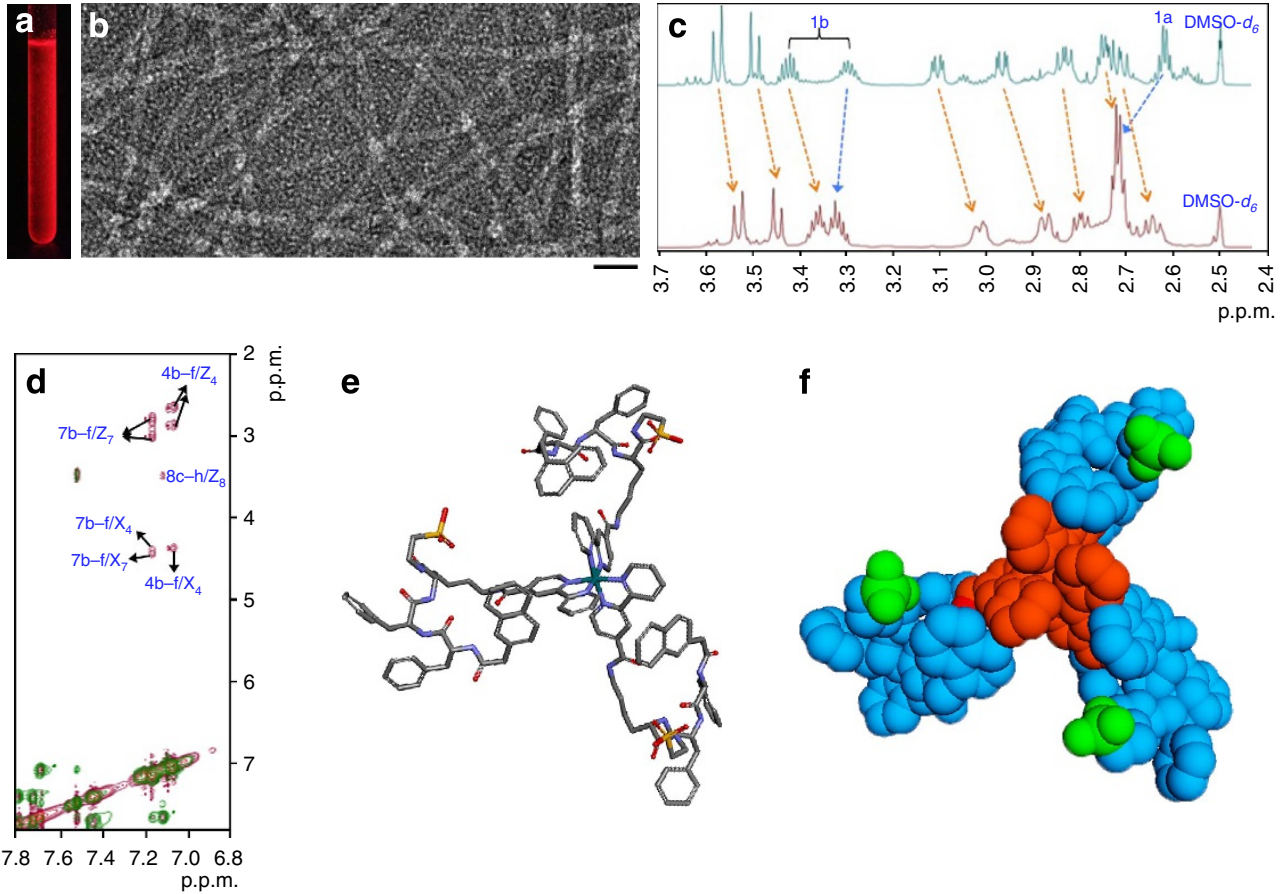

Figure 4 | 3D molecular structure of $\mathbf{5}$ in DMSO and $\mathbf{D}_{\mathbf{2}} \mathbf{O}$ mixture based on 2D NMR spectra. (a) Optical image of $\mathbf{5}$ in $D M S O-d_{6} / D_{2} O(2 / 1)$ in NMR tube under Ultraviolet light (on the right of the NMR tube). (b) HRTEM of the mixture in NMR tube of Figure a. (c) ${ }^{1} \mathrm{H}$ NMR spectra of $\mathbf{5}$ in DMSO (cyan) and in DMSO/D $\mathrm{D}_{2} \mathrm{O}\left(2 / 1\right.$; dark red) indicate the upfield shift of protons close to sulfonate. (d) Overlapped spectra of ${ }^{1} \mathrm{H}-\mathrm{TOCSY}$ (green) and ${ }^{1} \mathrm{H}-\mathrm{NOESY}$ (red) with assignment of NOEs. (e) Stick model and (f) space-filling model (colour representation is same as in Fig. 2) of $\mathbf{5}$ in DMSO- $d_{6} / \mathrm{D}_{2} \mathrm{O}$. 
conformers under conditions of self-assembly, the decreased spectral dispersion and increased degeneracy of diastereotopic proton signals (Fig. 4c) indicates that discrete folded conformations are less favoured under these conditions. Combining this information with excluded volumes due to steric interactions, we propose that trefoil 5 adopts an unfolded conformation (Fig. 4e,f) with maximum length of $34.2 \AA$ under conditions that lead to self-assembly of single molecule stacking for fibrils with width of $4.5 \pm 0.1 \mathrm{~nm}^{31}$. We also observed upfield shifts of protons (1a and 1 b; Fig. 4c) in the vicinity of the sulfonate groups and downfield shifts of all the other protons (Supplementary Fig. 4) after introducing $\mathrm{D}_{2} \mathrm{O}$ into the system. These shifts agree with the unfolding of 5 .

Self-assembly and $\mathrm{pH}$ modulation. On the basis of the unfolded 3D structure of $\mathbf{5}$ in the mixed aqueous medium, multiple forces potentially contribute to the self-assembly of $\mathbf{5}$, including hydration, electrostatics and hydrophobic interactions. These various contributions can be modulated by altering the $\mathrm{pH}$. The sulfonate groups of $\mathbf{5}$ are ionisable and exist predominantly in the negatively charged form $\left(-\mathrm{SO}_{3}^{-}\right)$at $\mathrm{pH}$ values above the $\mathrm{p} K_{\mathrm{a}}$. The hydration of the sulfonate group obviously depends on its ionization state (that is, the sulfonate anion is more strongly hydrated than the neutral sulfonic $\operatorname{acid}^{37,38}$ ). Thus, changing $\mathrm{pH}$ will effectively modulate both the hydration and electrostatics of 5 .

We evaluated the hydrogelation of $5(4 \mathrm{mM})$ in water from $\mathrm{pH}$ 14 to 1 and use transmission electron microscopy (TEM) to examine the nanostructures formed. As shown in Fig. $5 \mathrm{a}, 5$ is able to form red hydrogels at basic to neutral $\mathrm{pH}$. Acidic $\mathrm{pH}$ condition causes syneresis, with collapse of the hydrogel and eventual suspension. The decrease in the water-carrying capacity of $\mathbf{5}$ under conditions of low $\mathrm{pH}$ suggests an increase in the intermolecular hydrophobic interactions. The circular dichroism spectra (Supplementary Fig. 9) of $5(4 \mathrm{mM})$ in water indicate the beta-sheet formation at basic to acidic $\mathrm{pH}$, and higher $\mathrm{pH}$ favours beta-sheet formation.

The morphologies of the nanostructures revealed by the TEM images (Fig. 5a) support the hydrogelation behaviours of $\mathbf{5}$ in water as a function of $\mathrm{pH}$. The TEM images obtained as a function of gelation time also reveals a two-stage cooperativity 39 in the self-assembly of $\mathbf{5}$. Initially, self-assembly of $\mathbf{5}$ in water results in similar uniform fibrils with $5 \pm 1 \mathrm{~nm}$ widths regardless of $\mathrm{pH}$ (Supplementary Fig. 7). The $\mathrm{pH}$-dependent transformation of nanofibres to other morphologies occurs after an incubation period (for example, $48 \mathrm{~h}$; Supplementary Fig. 8). At pH 10, the self-assembled fibrils observed after aging are the same as those seen initially, so no secondary rearrangement occurs. At $\mathrm{pH} 7$, the fibrils maintain a uniform width but become shorter than the fibrils observed initially (or after aging at $\mathrm{pH} 10$ ). Some fibrils append to each other, indicating increased interfibrillar interactions. At $\mathrm{pH} 4$, the nanofibres transform into rectangular lamellar structure with $19-87 \pm 1 \mathrm{~nm}$ in width and a few micrometres in length. When the $\mathrm{pH}$ reaches 1 , the separated uniform nanofibres transform into intertwined fibrils bundles. Apparently, the uniform nanofibres are the kinetically favoured morphology at the initial stage of self-assembly of 5 . The $\mathrm{pH}$ dependent morphologies reached after aging appear to be thermodynamically stable.

The uniform long fibrils formed under basic conditions are more flexible than other self-assembled morphologies of $\mathbf{5}$ at neutral or acidic $\mathrm{pH}$. Since the fibrils are negatively charged under the basic conditions, the interfibrillar repulsive interactions favour thin split nanofibrils, which results in a softer hydrogel. As the $\mathrm{pH}$ decreases, the sulfonate groups are progressively protonated $\left(-\mathrm{SO}_{3} \mathrm{H}\right)$, decreasing interfibrillar repulsion, thus allowing greater interfibrillar interaction that contributes to the distribution of less tunable (shorter) fibrils and increased interfibrillar contact, as revealed in the TEM images (Fig. 5a). At $\mathrm{pH} 4$, the collapse of the gel agrees with the transformation of fibrils to lamellar structures (Fig. 5a). The formation of such morphology leads to a significant reduction of the surface that is exposed to the aqueous medium compared with the nanofibres. At $\mathrm{pH} 1$, nanofibres transform into the most compact morphology observed. Decreases in both the surface area available for solvent interactions and the corresponding hydration of the surface due to increased intermolecular hydrophobic interactions likely contribute to the syneresis of the hydrogel at

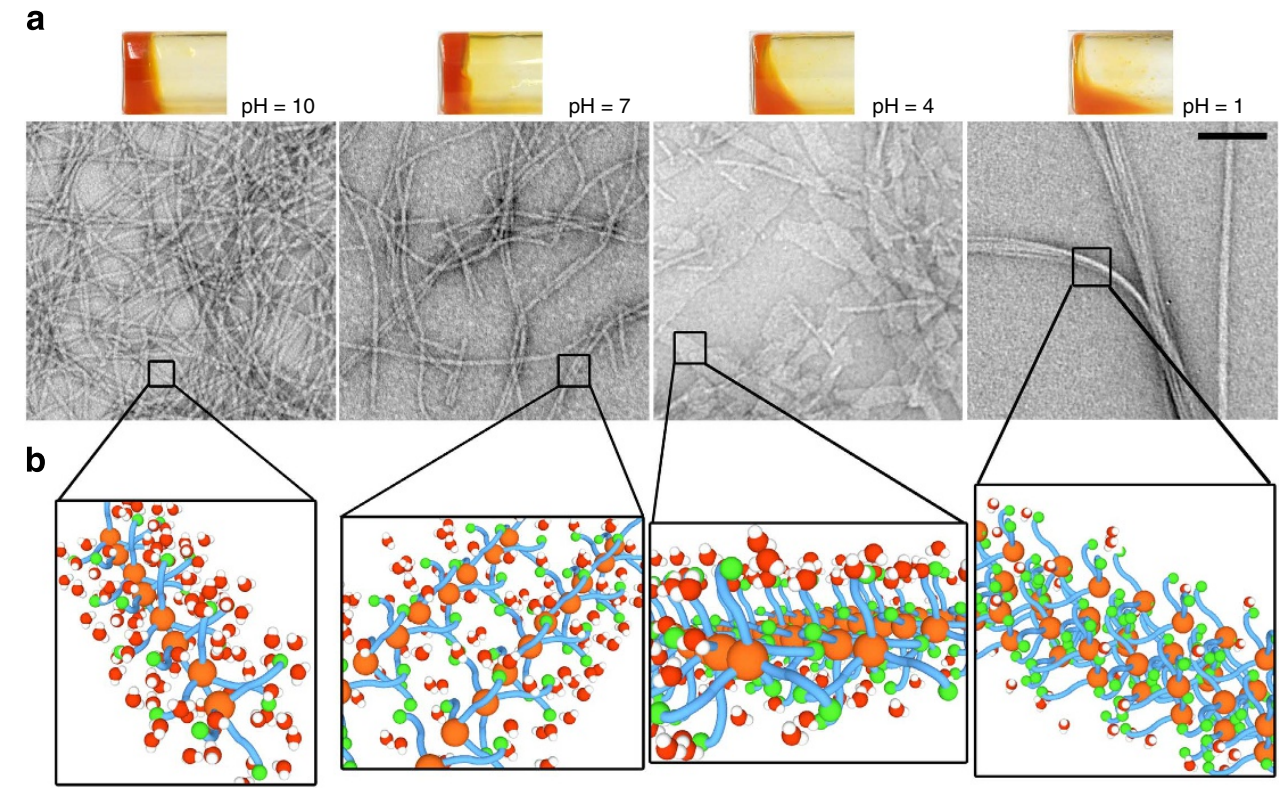

Figure $\mathbf{5}$ | The morphological change of the assemblies of $\mathbf{5}$ upon pH modulation. (a) Optical images and TEM images of $\mathbf{5}$ in water (4 mM) at different $\mathrm{pH}$ with morphology change (scale bar, $100 \mathrm{~nm}$ ). (b) Illustration of the enhanced hydration level and negative charges of sulfonate groups by increasing $\mathrm{pH}$ results in multiple self-assembled morphologies. 
low $\mathrm{pH}$. These results illustrate that changing $\mathrm{pH}$ can modulate the interactions between fibrils by perturbing the balance between the electrostatic repulsion and hydrophobic interactions, thus resulting in different dominant morphologies at different $\mathrm{pH}$ values (Fig. 5b). This observation agrees with the mechanism elucidated by Boden et al. ${ }^{12,13}$, that is, multilevel of intermolecular and interfibrillar interactions dictate the morphology of the self-assembled nanostructures.

\section{Discussion}

Although bottom-up molecular engineering has been extensively applied in biomimetic design, the execution of polymorphism through an integrated molecular system still remains as a great challenge in materials science. To achieve this goal, fully understanding not only the individual building blocks but also the path from simplicity to complexity and efficient manipulation of molecular structure are required. By describing the design and synthesis of a trefoil zwitterionic metallopeptide that mimics the conformational transitions of proteins, we present a successful case of biomimetic folding dynamics of proteins. The elucidation of conformation transition and polymorphic self-assembly of the molecule through conventional techniques, 2D NMR, and TEM offers molecular-level insights on mechanisms of formation of self-assembled nanostructures. This research as a contribution to the exploration of polymorphism of molecular arrangements will provide insights for understanding the multiple molecular interactions that associated with the disease-causing amyloids. The polymorphic self-assembly of the trefoil molecule by $\mathrm{pH}$ modulation will also contribute to the understanding of the heterogeneous $\mathrm{pHs}$ in a cellular setting for producing polymorphic aggregates of proteins and peptides. Overall, this study provides valuable information on designing single molecular systems for exploring the conformational switch with high precision $^{40}$.

\section{Methods \\ NMR spectroscopy. The NMR sample of $\mathbf{5}$ without self-assembly was prepared by adding $10 \mathrm{mg}$ of 5 and $500 \mu \mathrm{l}$ DMSO- $d_{6}$ into an NMR tube and mixing them well. The NMR sample of $\mathbf{5}$ with self-assembly was prepared by dissolving $10 \mathrm{mg}$ of $\mathbf{5}$ in $333 \mu \mathrm{l} \mathrm{DMSO}-d_{6}$ in an NMR tube NMR tube, followed by the addition of $167 \mu \mathrm{l}$ $\mathrm{D}_{2} \mathrm{O}$ for well mixing. The NMR spectra were recorded on a Bruker Avance NMR spectrometer operating at $800.13 \mathrm{MHz}\left({ }^{1} \mathrm{H}\right)$ and equipped with TCI cryoprobe and pulse-field gradients. Experiments used for sequential resonance assignments include ${ }^{1} \mathrm{H}$-TOCSY, and ${ }^{1} \mathrm{H}$-NOESY. All experiments were performed at $298 \mathrm{~K}$. NMR data were processed using TOPSPIN (Bruker Biospin Inc.) and the data were analysed using TOPSPIN and MestReNova.}

Gelation test. A small glass vial was charged with $5.73 \mathrm{mg}$ compound $\mathbf{5}$ and $500 \mu \mathrm{l}$ deionized water. The mixture was sonicated for dissolving. Trace amount of concentrated nitric acid or sodium hydroxide solution was added into the mixture for $\mathrm{pH}$ modulation. The final mixture was sonicated until mixed well.

High-resolution transmission electron microscopy. HRTEM micrographs were obtained with TITAN 80-300 FEG scanning transmission electron microscope by the negative-staining method with uranyl acetate solution $(2 \%)$ as the staining reagent.

Transmission electron microscopy. TEM images were acquired by conventional TEM technique. The specimen was prepared by placing a droplet of solution/ hydrogel of $\mathbf{5}$ onto a holey carbon-coated copper grid. After negative-stained with uranyl acetate solution (2\%), the grid was allowed to air dray. TEM characterization was performed using a Morgani 268 transmission electron microscope.

\section{References}

1. Ellis, R. J. The "bio" in biochemistry: protein folding inside and outside the cell. Science 272, 1448-1449 (1996).

2. Gething, M. J. \& Sambrook, J. Protein folding in the cell. Nature 355, 33-45 (1992).

3. Horwich, A. L. Protein folding in the cell: an inside story. Nat. Med. 17, 1211-1216 (2011).
4. Kelly, J. W. \& Balch, W. E. The integration of cell and chemical biology in protein folding. Nat. Chem. Biol. 2, 224-227 (2006).

5. Si, K., Lindquist, S. \& Kandel, E. R. A neuronal isoform of the aplysia CPEB has prion-like properties. Cell 115, 879-891 (2003).

6. Prusiner, S. Molecular biology of prion diseases. Science 252, 1515-1522 (1991).

7. Colby, D. W. \& Prusiner, S. B. De novo generation of prion strains. Nat. Rev. Microbiol. 9, 771-777 (2011)

8. Pepys, M. B. et al. Human lysozyme gene mutations cause hereditary systemic amyloidosis. Nature 362, 553-557 (1993).

9. Hurle, M. R., Helms, L. R., Li, L., Chan, W. \& Wetzel, R. A role for destabilizing amino acid replacements in light-chain amyloidosis. Proc. Natl Acad. Sci. USA 91, 5446-5450 (1994).

10. Gustafsson, L., Leijonhufvud, I., Aronsson, A., Mossberg, A. \& Svanborg, C. Treatment of skin papillomas with topical alpha-lactalbumin-oleic acid. New Engl. J. Med. 350, 2663-2672 (2004).

11. Perriman, A. W. et al. Reversible dioxygen binding in solvent-free liquid myoglobin. Nat. Chem. 2, 622-626 (2010).

12. Aggeli, A. et al. Hierarchical self-assembly of chiral rod-like molecules as a model for peptide beta-sheet tapes, ribbons, fibrils, and fibers. Proc. Natl Acad. Sci. USA 98, 11857-11862 (2001).

13. Aggeli, A. et al. Responsive gels formed by the spontaneous self-assembly of peptides into polymeric beta-sheet tapes. Nature 386, 259-262 (1997).

14. Ulijn, R. V. \& Caponi, P. F. Bioinspired chemistry rewiring nanostructures. Nat. Chem. 2, 521-523 (2010).

15. Liang, G. L., Ren, H. J. \& Rao, J. H. A biocompatible condensation reaction for controlled assembly of nanostructures in living cells. Nat. Chem. 2, 54-60 (2010)

16. Lv, G. H. et al. Structural comparison of mouse and human alpha-synuclein amyloid fibrils by solid-state NMR. J. Mol. Biol. 420, 99-111 (2012).

17. Nelson, R. \& Eisenberg, D. Recent atomic models of amyloid fibril structure. Curr. Opin. Struc. Biol. 16, 260-265 (2006).

18. Nelson, R. et al. Structure of the cross-beta spine of amyloid-like fibrils. Nature 435, 773-778 (2005).

19. Sawaya, M. R. et al. Atomic structures of amyloid cross-beta spines reveal varied steric zippers. Nature 447, 453-457 (2007).

20. Makin, O. S., Atkins, E., Sikorski, P., Johansson, J. \& Serpell, L. C. Molecular basis for amyloid fibril formation and stability. Proc. Natl Acad. Sci. USA 102, 315-320 (2005).

21. Ben-Naim, A. Solvent-induced forces in protein folding reflections on the protein folding problem. Curr. Opin. Colloid. In. 18, 502-509 (2013).

22. Creighton, T. E. Toward a better understanding of protein folding pathways. Proc. Natl Acad. Sci. USA 85, 5082-5086 (1988).

23. Némethy, G., Peer, W. J. \& Scheraga, H. A. Effect of protein-solvent interactions on protein conformation. Ann. Rev. Biophys. Bioeng. 10, 459-497 (1981).

24. Anfinsen, C. B. Principles that govern folding of protein chains. Science 181, 223-230 (1973).

25. Anfinsen, C. B. \& Acherage, H. A. Experimental and Theoretical Aspects of Protein Folding 29 (Academic Press, Inc., 1975).

26. Ringe, D. \& Petsko, G. A. How enzymes work. Science 320, 1428-1429 (2008).

27. Mitchell, S. G. et al. Face-directed self-assembly of an electronically active Archimedean polyoxometalate architecture. Nat. Chem. 2, 308-312 (2010).

28. Lovell, J. F. et al. Porphysome nanovesicles generated by porphyrin bilayers for use as multimodal biophotonic contrast agents. Nat. Mater. 10, 324-332 (2011).

29. Kuroiwa, K. et al. Self-Assembly of tubular microstructures from mixed-valence metal complexes and their reversible transformation by external stimuli. Angew. Chem. Int. Edit. 51, 656-659 (2012).

30. Kuwahara, R., Fujikawa, S., Kuroiwa, K. \& Kimizuka, N. Controlled polymerization and self-assembly of halogen-bridged diruthenium complexes in organic media and their dielectrophoretic alignment. J. Am. Chem. Soc. 134, 1192-1199 (2012).

31. Zhang, Y., Kuang, Y., Gao, Y. A. \& Xu, B. Versatile small-molecule motifs for self-assembly in water and the formation of biofunctional supramolecular hydrogels. Langmuir 27, 529-537 (2011).

32. Kuang, Y., Gao, Y., Shi, J. F., Li, J. \& Xu, B. The first supramolecular peptidic hydrogelator containing taurine. Chem. Commun. 50, 2772-2774 (2014).

33. Burstall, F. H. Optical activity dependent on co-ordinated bivalent ruthenium. J. Chem. Soc. 173-175 (1936).

34. Ford, P. C. Properties and reactions of ruthenium(ii) amine complexes. Coord. Chem. Rev. 5, 75-99 (1970).

35. Guo, J., Mayers, P. C., Breault, G. A. \& Hunter, C. A. Synthesis of a molecular trefoil knot by folding and closing on an octahedral coordination template. Nat. Chem. 2, 218-222 (2010)

36. Biner, M., Burgi, H. B., Ludi, A. \& Rohr, C. Crystal and molecular-structures of $\left[\mathrm{Ru}(\mathrm{Bpy})_{3}\right]\left(\mathrm{Pf}_{6}\right)_{3}$ and $\left[\mathrm{Ru}(\mathrm{Bpy})_{3}\right]\left(\mathrm{Pf}_{6}\right)_{2}$ at 105 K. J. Am. Chem. Soc. 114, $5197-$ 5203 (1992).

37. Clark, J. K., Paddison, S. J., Eikerling, M., Dupuis, M. \& Zawodzinski, T. A. A comparative $\mathrm{ab}$ initio study of the primary hydration and proton dissociation of various imide and sulfonic acid ionomers. J. Phys. Chem. A 116, 1801-1813 (2012). 
38. Selvan, M. E., Calva-Munoz, E. \& Keffer, D. J. Toward a predictive understanding of water and charge transport in proton exchange membranes. J. Phys. Chem. B 115, 3052-3061 (2011).

39. Mirny, L. \& Shakhnovich, E. Protein folding theory: from lattice to all-atom models. Annu. Rev. Biophys. Biomol. Struct. 30, 361-396 (2001).

40. Wei, B., Dai, M. \& Yin, P. Complex shapes self-assembled from single-stranded DNA tiles. Nature 485, 623-626 (2012).

\section{Acknowledgements}

This work was supported by NSF MRSEC grant (DMR-0820492) and W.M. Keck Foundation.

\section{Author contributions}

Y.Z. and B.X. wrote the manuscript. Y.Z. and B.X. designed the molecule and the experiments. Y.Z. synthesized the molecules, analysed data and prepared the figures.
S.S.P. and T.C.P. provided invaluable advice and assisted with the NMR experiments and the manuscript preparation. N.Z. assisted with the synthesis and data analysis. J.S assisted with the TEM tests. B.Z. and X.Z. performed the HRTEM tests.

\section{Additional information}

Supplementary Information accompanies this paper at http://www.nature.com/ naturecommunications

Competing financial interests: The authors declare they have no competing financial interests.

Reprints and permission information is available online at http://npg.nature.com/ reprintsandpermissions/

How to cite this article: Zhang, Y. et al. Unfolding a molecular trefoil derived from a zwitterionic metallopeptide to form self-assembled nanostructures. Nat. Commun. 6:6165 doi: $10.1038 /$ ncomms7165 (2015) 